\title{
An Empirical Analysis of the Price Discovery Function of Dalian Soybean Futures Market
}

\author{
Xiang Yi ${ }^{1, a}$, Sun Qiming ${ }^{1}$, Sun Yin ${ }^{1}$, Li Kai ${ }^{1, b^{*}}$ \\ ${ }^{1}$ Beijing University of Science and Technology, Beijing, China \\ ayixiang@xs.ustb.edu.cn, blikai2000@ustb.edu.cn \\ ${ }^{*}$ Corresponding Author
}

\begin{abstract}
Using the daily closing prices of the soybean futures in Dalian future markets and the average price of the soybean spots in different provinces in China from December 1th.2014 to April 30th.2015 , this paper analyzes the role of Dalian soybean futures by applying correlation analyses, unit root test, co-integration test(Kao,Johansen,E-G), Granger causality test, error correction model,impulse response and variance decomposition. The results of such models and analysis indicate that a strong relationship between the spot price of soybean and the future price of its exists. In other words, an effective price discovery function is available in Dalian soybean future market.
\end{abstract}

Key words: soybean futures, Price discovery,Granger causality test,causality,impulse response

$$
\begin{aligned}
& \text { 我国大豆期货与现货价格关系的实证研究 } \\
& \text { 项翊 }{ }^{1, \mathrm{a}} \text {, 孙启明 }{ }^{1} \text {, 孙颖 }{ }^{1} \text {, 李凯 }{ }^{1, \mathrm{~b}^{*}} \\
& { }^{1} \text { 北京科技大学东凌经济管理学院, 北京, 中国 } \\
& \text { ayixiang@xs.ustb.edu.cn, blikai2000@ustb.edu.cn } \\
& \text { *通讯作者 }
\end{aligned}
$$

中文摘要.本文选取2014年12月1日至2015 年4 月30日期间中国大豆期货与现货市场 102 个交易日的价格数据, 运用ADF单位根检 验、协整检验(Kao检验, Johansen协整检验, E-G两步法）、Granger 因果检验、误差修正 模型、脉冲响应函数和方差分解分析对我国 大豆期货与现货价格数据之间引导关系进行 实证分析，发现期货和现货价格偏离时都能 在短期内趋近于长期均衡, 但总体呈现期货 价格对现货价格的单项引导关系。由此看来 我国大豆期货市场形成13年来已经初步具备 价格发现的功能。

关键词：大豆期货；价格发现；格兰杰因果 检验; 协整检验; 脉冲响应函数

\section{1. 引言}

本文现货价格选取的是从2014年12月1 日至2015年4月30日全国各省份大豆当日售 价的平均值, 来源于芝华数据, 能够很好得 反映我国大豆现货市场价格的水平及其变 动, 用 XH表示。期货价格选取的是同时期大 连期货交易所每日收盘价, 来源于大连期货 交易所的日交易数据用 $\mathrm{QH}$ 表示, 选取的价格 为期货主力期货价格，2014年12月至2015年2 月采用的是豆二 1505 (B1505)的每日结算价, 2015年3月至4月采用的是豆二1509（B509） 的每日结算价。为了增强数据的稳定性, 对 期货价格与现货价格取对数, 分别记为 $\operatorname{lnqh}, \ln \mathrm{xh}^{[1]}$ 。 


\section{2.期货价格以及现货价格趋势分析}

从2014年12月开始，大豆价格经历了先 上涨再下降, 后又上涨的趋势。可以看出 2014 年12月至2015年1月底, 期现货价格长期趋势 上涨, 而且期货有带动现货上涨的趋势, 而 2014年2月至4月底则是价格先降后涨，并且 期货价格也是领先于现货，从画出的折线图 可以看出期现货价格基本趋势相同 ${ }^{[1]}$ 。并且 计算出的相关系数为 $0.8562>0.8$, 属于强相 关。然而相关性只是统计特性, 还需要进一 步的验证证明期货与现货之间的关系。

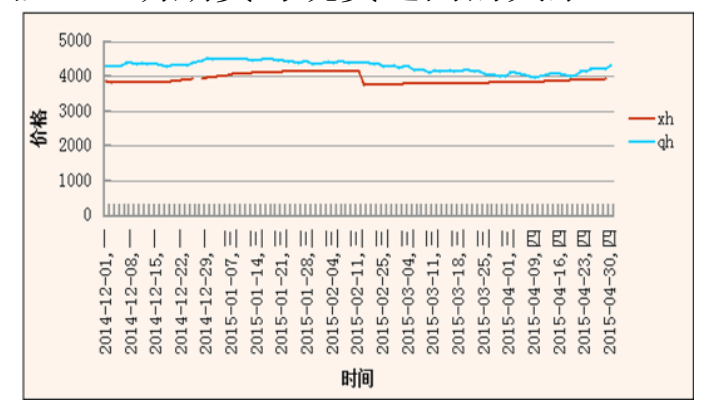

图一.期货价格与现货价格走势

\section{ADF单位根检验}

为了避免虚假回归，在协整检验之前需 要进行ADF单位根检验, 用于检验数据是否 平稳, 根据 $\mathrm{AIC}$ 原则确定滞后阶数。对期货 与现货价格的对数进行检验的结果表明, 在 $1 \%$ 与 $5 \%$ 的显著性水平下原假设都是存在的， 即存在单位根, 意味着 $\operatorname{lnqh}$ 与 $\ln x h$ 是非平稳 的。对期货与现货价格对数的一阶差分进行 检验的结果表明 $1 \%$ 与 $5 \%$ 的显著性水平下原 假设是不存在的, 即不存在单位根, 意味着 $\triangle \operatorname{lnxh}$ 与 $\triangle \operatorname{lnqh}$ 是平稳的 ${ }^{[3]}$ 。因此 $\operatorname{lnxh}$ 与 $\operatorname{lnqh}$ 是一阶单整的, 表示为I (1), 因此可以进 一步进行协整检验。

表1.期货价格与现货价格ADF单位根检验

\begin{tabular}{|c|c|c|c|c|}
\hline 变量 & ADF值 & 1\%临界值 & $\mathbf{5 \%}$ 临界值 & 平稳 \\
\hline $\ln x h$ & -1.594 & -4.059 & -3.458 & 否 \\
\hline $\ln q h$ & -1.538 & -4.056 & -3.457 & 否 \\
\hline$\triangle \ln x h$ & -9.865 & -4.064 & -3.461 & 是 \\
\hline$\triangle \ln q h$ & -8.975 & -4.061 & -3.459 & 是 \\
\hline
\end{tabular}

\section{4.协整检验}

协整是对非平稳经济变量长期均衡关系 的统计描述, 存在协整关系的非平稳变量的 非均衡误差是平稳。本文采用Kao检验 johansen协整检验以及EG检验对大豆期货和 现货价格进行协整检验。Kao检验的结果表 明, 在 $5 \%$ 的置信水平下ADF检验统计量显 著，拒绝没有协整关系的原假设，因此kao检 验认为我国大豆期货和现货价格之间存在协 整关系。从显著性为 0.10 的johansen协整检验 可以看出期现货的 $\ln x h$ 以及 $\operatorname{lnqh}$ 在 $10 \%$ 置信 度水平下拒绝了不存在协整关系的原假设， 意味着存在协整关系, 因此期货价格与现货 价格之间是协整的。

表2.kao检验.Johansen协整检验.E-G两步法结果

\begin{tabular}{|l|c|c|c|c|}
\hline 检验名称 & 原假设 & t统计量 & $\mathbf{P}$ 值 & $\begin{array}{c}\text { 协整 } \\
\text { 关系 }\end{array}$ \\
\hline Kao检验 & $\begin{array}{c}\text { 不存在 } \\
\text { 协整关系 }\end{array}$ & -2.081654 & 0.0187 & 存在 \\
\hline $\begin{array}{l}\text { Johansen } \\
\text { 协 整 检 } \\
\text { 验 }\end{array}$ & $\begin{array}{c}\text { 不存在 } \\
\text { 协整关系 }\end{array}$ & 14.25930 & 0.0761 & 存在 \\
\hline & $\begin{array}{l}\text { 最多存 } \\
\text { 在一个 }\end{array}$ & 3.668621 & 0.0827 & 存在 \\
\hline $\begin{array}{l}\text { E-G两 } \\
\text { 步法 }\end{array}$ & $\begin{array}{l}\text { 不存在 } \\
\text { 协整 }\end{array}$ & -2.006171 & 0.0435 & 存在 \\
\hline
\end{tabular}

再进行E-G两步法检验，第一步：首先以 lnxh为被解释变量, lnqh为解释变量进行线性 回归, 得到公式:

$\ln x h=3.396381+0.583272 \ln q h+e$

随后导出残差 $\mathrm{e}$ 。第二步: 对残差e进行 $\mathrm{ADF}$ 单位根检验。检验结果数据 $\mathrm{p}=0.0435$ 表 明拒绝原假设, 不存在单位根, 即残差 $\mathrm{e}$ 是平 稳的。当残差 $\mathrm{e}$ 平稳时代表着现货价格与期货 价格是协整的。上面三种协整检验均表明 $\ln x h$ 与 $\ln q h$ 是协整的, 即期货价格与现货价格 之间存在着内在的传导机制。

\section{Granger 因果检验}

大豆价格发现功能的实现程度可通过大 豆期货价格和现货价格之间的关系来判断。 如果期货市场具有价格发现功能, 则期货价 格应该为该价格对应合约到期日现货价格的 无偏估计量, 因此需要检验期货价格是否对 现货价格具有引导作用。大豆期货和大豆现 
货价格之间存在协整关系只能说明两变量间 存在长期均衡关系，但是期货与现货价格之 间是否存在因果关系还需要进一步检验。选 择利用格兰杰因果检验进行因果关系分析, 通过LM检验可以看出滞后 1 期时随机干扰项 序列不相关而滞后两阶后随机干扰项序列相 关, 因此在检验时选取滞后一阶。检验结果 如下表。

表3.格兰杰因果检验结果

\begin{tabular}{|c|l|c|c|}
\hline 零假设 & F统计量 & P值 & 结论 \\
\hline $\begin{array}{c}\mathrm{QH} \text { 不是 } \mathrm{XH} \text { 的 } \\
\text { 格兰杰原因 }\end{array}$ & 3.16965 & 0.0423 & $\begin{array}{c}\mathrm{QH} \text { 是 } \mathrm{XH} \text { 的 } \\
\text { 格兰杰原因 }\end{array}$ \\
\hline $\begin{array}{c}\mathrm{XH} \text { 不是 } \mathrm{QH} \text { 的 } \\
\text { 格兰杰原因 }\end{array}$ & 2.64981 & 0.0695 & $\begin{array}{c}\mathrm{XH} \text { 是 } \mathrm{QH} \text { 的 } \\
\text { 格兰杰原因 }\end{array}$ \\
\hline
\end{tabular}

由上表显示, $\mathrm{F}$ 统计量在 $5 \%$ 的显著性水 平下拒绝了 $\mathrm{QH}$ 不是 $\mathrm{XH}$ 的格兰杰原因的原假 设, 说明期货价格是现货价格的格兰杰原因; $\mathrm{F}$ 统计量在 $5 \%$ 的显著性水平下接受了 $\mathrm{XH}$ 不 是 $\mathrm{QH}$ 的格兰杰原因的原假设, 说明现货价格 不是期货价格的格兰杰原因。因此大豆期货 价格是现货价格的格兰杰原因而现货价格不 是期货价格的格兰杰原因，即期货和现货市 场价格存在期货价格引导现货价格的单向因 果和引导关系

\section{6. 误差修正模型(ECM)}

协整模型是用来考察变量间的长期关 系，而误差修正模型体现的是变量间的短期 变动关系。因此将协整模型求出的残差 $\mathrm{e}$ (或 称 $\mathrm{ecm}$ )带入误差修正模型进行构建, 由AIC 准则和SC准则决定滞后 1 阶, 用OLS 在协整的 基础上建立模型:

$$
\begin{aligned}
& d \ln x h=c+\beta_{1} d \ln q h+\beta_{2} d \ln q h(-1) \\
& +\beta_{3} d \ln x h(-1)+\lambda e c m(-1)+\mu
\end{aligned}
$$

由此可得到模型:

$d \ln x h=0.470291+0.809270 d \ln q h+0.691837$ $d \ln q h(-1)-0.247196 d \ln x h(-1)-0.371027 e c$ $m(-1)$
表4.误差修正模型输出结果

Dependent Variable: DLNXH

Method: Least Squares

Date: 06/08/15 Time: 12:53

Sample (adjusted): 12/04/2014 4/29/2015

\begin{tabular}{|c|c|c|c|c|}
\hline & $\begin{array}{r}\text { Coeffi } \\
\text { cient }\end{array}$ & Std. Error & $\begin{array}{r}\mathrm{t} \text {-Statist } \\
\text { ic }\end{array}$ & Prob. \\
\hline C & $\begin{array}{c}0.470 \\
291 \\
0809\end{array}$ & 0.001131 & $\begin{array}{c}-0.0845 \\
91 \\
28984\end{array}$ & 0.9328 \\
\hline DLNQH & $\begin{array}{l}270 \\
0.691\end{array}$ & 0.135468 & $\begin{array}{c}28.984 \\
47 \\
4.8294\end{array}$ & 0.0000 \\
\hline DLNQH(-1) & $\begin{array}{c}837 \\
-0.24\end{array}$ & 0.134763 & $\begin{array}{c}75 \\
6.2849\end{array}$ & 0.0392 \\
\hline DLNXH(-1) & $\begin{array}{l}7196 \\
-0.37\end{array}$ & 0.109939 & $\begin{array}{c}35 \\
2.2420\end{array}$ & 0.0193 \\
\hline$E(-1)$ & 1027 & 0.043198 & 38 & 0.0476 \\
\hline & 0.802 & \multirow{6}{*}{\multicolumn{2}{|c|}{$\begin{array}{c}\text { Mean dependent } \\
\text { var } \\
\text { S.D. dependent } \\
\text { var } \\
\text { Akaike info } \\
\text { criterion }\end{array}$}} & 5.00E-0 \\
\hline R-squared & 743 & & & 5 \\
\hline Adjusted & 0.792 & & & 0.01066 \\
\hline R-squared & 741 & & & \\
\hline S.E. of & 0.010 & & & -6.2029 \\
\hline regression & 588 & & & 65 \\
\hline Sum squared & 0.009 & \multirow{2}{*}{\multicolumn{2}{|c|}{$\begin{array}{c}\text { Schwarz criterion } \\
\text { Hannan-Quinn }\end{array}$}} & -6.0622 \\
\hline resid & $\begin{array}{l}306 \\
277.9\end{array}$ & & & $\begin{array}{c}07 \\
-6.1462\end{array}$ \\
\hline Log likelihood & 305 & \multirow{3}{*}{\multicolumn{2}{|c|}{$\begin{array}{l}\text { criter. } \\
\text { Durbin-Watson } \\
\text { stat }\end{array}$}} & 57 \\
\hline & 146.9 & & & 1.88840 \\
\hline F-statistic & $\begin{array}{l}285 \\
0.000\end{array}$ & & & 0 \\
\hline Prob(F-statistic) & 000 & & & \\
\hline
\end{tabular}

Included observations: 88 after adjustments

可以看出, 误差修正模型通过F检验以及 $\mathrm{T}$ 检验, 说明变量总体和个体都显著, 并且相 关系数达到 0.8 , 相关性较强。当期货价格变 动 1 个点位的时候期货变动 0.8 个点, 期货滞 后一期价格变动 1 时期货现期价格变动 0.6 , 说明期货的价格传导功能在同一时期造成的 影响更大。因此期货价格对现货价格的价格 传导机制较为有效。

\section{7. 脉冲响应函数分析}

脉冲响应函数能有效描述大豆期现货价 格分别受到自身以及彼此的一个标准误差项 大小的冲击后当期值和未来值的变化程度, 结果如图二三所示, 其中响应长度选择50期。 图二为大豆现货市场对自身以及期货市场的 反应, 图三为大豆期货市场对自身以及现货 市场的反应。 


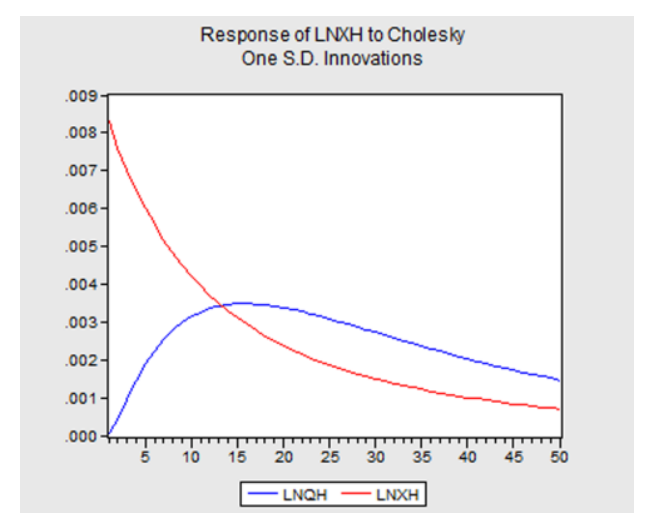

图二 大豆现货市场对自身以及现货市场的反应

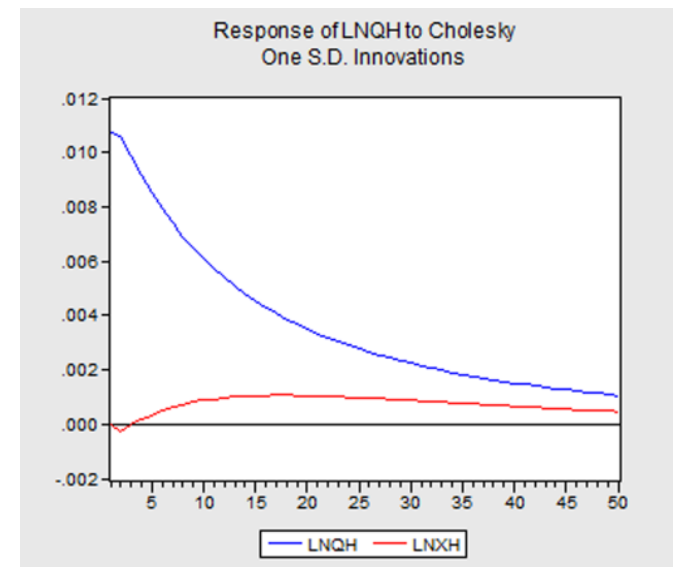

图三 大豆期货市场对自身以及现货市场的反应

从图二可以看出, 大豆现货价格对于自 身的一个标准差的信息反应灵敏 ${ }^{[5]}$, 第一期 最强的正向反应, 即来自自身的一个标准差 使价格上升了 $0.82 \%$, 随后反应呈现越来越缓 的下降趋势，到第 50 期时反应几乎为 $0.00 \%$ 。 大豆现货价格对于大豆期货价格的一个标准 差信息的冲击存在滞缓效应，第一期的反应 为 0 , 随后逐渐增加, 在第 17 期左右达到峰值 $0.38 \%$, 随后随着滞后期的增加（时间的拉 长），冲击逐渐消失。

从图三可以看出, 大豆期货价格对现货 价格的一个标准差新的冲击也存在滞缓效 应, 第一期反应为 0 , 随后虽逐渐增加, 但程 度并不明显, 峰值在 15 期左右达到 $0.1 \%$ 的正 向变化随后逐渐减少, 因此可以看出大豆期 货价格对现货价格的变化反应较弱。与此同 时, 大豆期货价格对其自身一个标准差的信 息反应灵敏，第一期具有最强的正向反应， 即来自自身的一个标准差使价格上升了 $1.1 \%$, 随后反应呈现越来越缓的下降趋势, 到第 50 期时反应几乎 $0.00 \%$ 。

由以上分析可以看出, 大豆期现货价格 分别对自身的一个标准差冲击呈现较强的反
应, 且该反应随着滞后期数的增加呈现减缓 下降。大豆现货市场对期货市场价格变动具 有滞后的反应, 且反应较强。而大豆期货市 场价格对现货市场的价格变动具有较弱的反 应。并且值得关注的是, 大豆现货市场在滞 后第13期左右受到的来自期货市场价格冲击 而产生的变化大于现货本身标准差冲击带来 的变化，而大豆期货市场一直到滞后50期都 是受到来自期货市场本身标准差冲击带来的 变化大, 由此可以看出价格发现功能偏于期 货价格引导现货价格的单向引导关系 ${ }^{[6]}$ 。

\section{8.方差分解分析}

方差分解是通过分析每一个结构冲击对 内生变量变化的贡献度来评价不同结构冲击 的重要性 ${ }^{[6]}$ 。在此本文进行对大豆期货价格 和现货价格 50期的方差分解, 结果图如下。

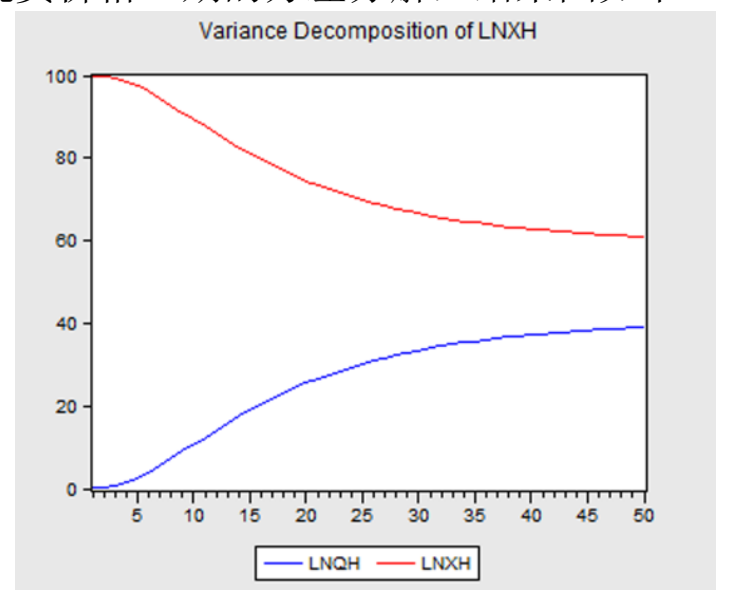

图四 大豆现货价格方差分解图

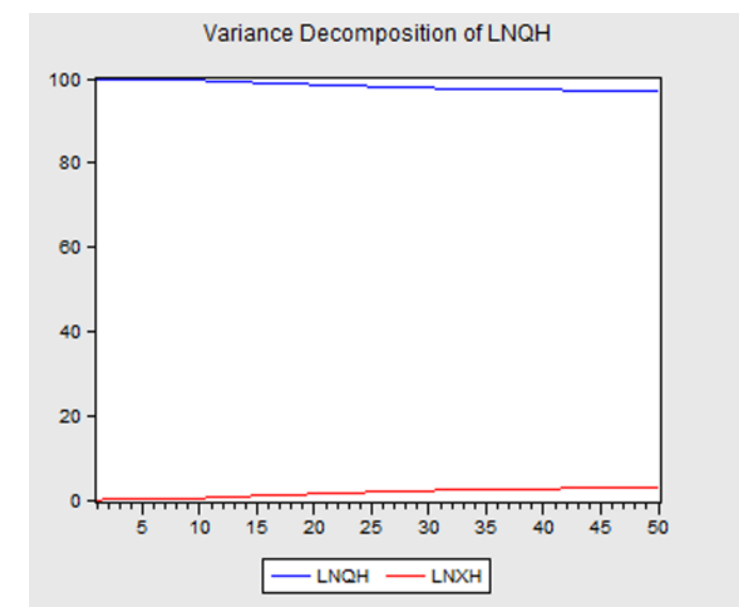

图五 大豆期货价格方差分解图

由大豆现货价格方差分解图可以看出, 第一期时大豆现货价格完全受自身影响, 此 后影响程度不断缓慢下降, 直到 50 期左右趋 于平稳, 影响程度为 $65 \%$ 左右。第一期时大 
豆现货价格受到期货价格微弱影响（1\%）, 此后影响程度不断增加并在 50 期左右趋于平 稳, 影响程度为 $39 \%$ 左右。

由大豆期货价格方差分解图可以看出, 从第一期到第 50 期, 期货价格受到自身价格 影响很大 (>95\%), 虽然随着滞后期的增加 略有减小，但程度极微小。期货价格受到现 货价格的影响有限 $(<5 \%)$, 且增加速度缓慢可 以忽略不计。

综上所述, 大豆现货市场受到期货市场 影响较大, 而期货市场受到现货市场影响有 限, 价格发现功能呈现出单向引导关系。

\section{9. 结束语}

价格发现是期货市场主要功能之一，可 以有效减少现货市场价格波动并且让期货市 场更加有效 ${ }^{[4]}$ 。本文分别在期现货市场上选 取了 88 个数据进行了实证分析, 采取的方法 分别是ADF单位根检验、协整检验 $(\mathrm{KaO}$ 检验, Johansen协整检验, E-G两步法)、Granger 因 果检验、误差修正模型, 脉冲响应函数和方 差分解分析, 检验结果表明我国大豆期货市 场具有期货市场引导现货市场的单向价格发 现功能, 主要结论如下。

（1）期货价格与现货价格是高度相关 的, 相关系数达到 0.8562 , 证明从统计特性 上来说存在长期的关系。

(2) 通过进一步的协整检验可以发现期 现货价格至少在 $10 \%$ 的显著性水平下是协整 的 (johansen检验结果), 而kao检验和E-G 两步法结果表明期现货价格在 $5 \%$ 的显著性 水平下都是协整的, 因此有足够的理由证明 期货价格与现货价格存在着长期的均衡关 系, 期货市场价格和现货市场价格即使出现 偏差或背离, 也会向趋于一致的方向发展, 这就为套期保值交易提供了前提条件。

(3) Granger 因果检验结果证明大豆期 货价格是现货价格的格兰杰原因, 而现货价 格不是期货价格的格兰杰原因, 即期货价格 有效引导现货价格, 期货和现货市场价格存 在单向因果和引导关系, 期货价格能够有效 反映现货价格未来变动方向的预期, 说明我 国大豆期货市场已经初步具备价格发现功能 [5]。

(4) 通过误差修正模型可以得出一个期 现货价格短期之间的变动关系, 从式 (3) 可
以看出, 当期货价格变动 1 个点位的时候现货 变动 0.8 个点, 期货滞后一期价格变动 1 时现 货现期价格变动 0.6 , 说明期货的价格传导功 能在同一时期造成的影响更大。

(5) 通过脉冲响应函数分析和方差分解 分析的结果可以看出我国大豆现货市场受到 期货市场影响较大，而期货市场受到现货市 场影响有限, 并且引导关系呈现先增加后减 弱的关系, 价格发现功能总体呈现出一定单 向引导关系。

根据以上分析可以得知, 大豆期货市场 存在着期货价格引导现货价格的单向因果和 引导关系, 并且影响随着滞后期的增加先增 大后减小。该结论表明我国大豆期货市场成 立13年来, 期货市场价格发现功能已经基本 具备。这也与实际生活中大豆期现货市场运 行多年, 已经可以有效地避免价格剧烈波动, 使得市场更加有效的事实相符。

\section{致谢}

本文受北京市青年英才计划（YETP0396） 资助。

\section{References}

[1] Liu lei,Price Discovery Function of China Cotton Future:Analysis Basing on The Comparison of American and China Cotton Future.Financial Theory \& Practice, 2010

[2]Yi Danhui,Data analysis and the apply of eviews, 2008

[3]Wang Zhen, Liu Zhenhai.Chen Chao.An Empirical Analysis of the Price Discovery Function of Shanghai Fuel Oil Futures Market,Petroleum Science. 2007

[4]SHIQING XIE and JIAJUN,HUANG.Price Discovery Function of Index Futures in China: Evidence from Daily Closing Prices. Economic and Political Studies.2013

[5]Li Kai, Zhang Chuanqi.An Empirical Analysis of the Relationship between the Price of Egg spots and of Egg futures,Financial Theory \& Practice, 2014

[6]Fang yan,Li xinxin, The mechanism of Price Conduction of China's Cotton Spots Markets and Future Markets,Financial Theory \& Practice, 2013 